\title{
MORTALIDADE POR DIABETES MELLITUS EM UMA MACRORREGIÃO DE SAÚDE DE MINAS GERAIS
}

\section{DIABETES MELLITUS MORTALITY ON A HEALTH MACRO-REGION OF MINAS GERAIS}

\section{LA MORTALIDAD POR DIABETES MELLITUS EN UNA MACRO-REGIÓN DE SALUD DE MINAS GERAIS}

\author{
Jéssica Mazutti Penso ${ }^{1}$, Eduardo Périco ${ }^{2}$
}

\begin{abstract}
RESUMO
Objetivo: analisar o padrão espaço-temporal da mortalidade por Diabetes Mellitus e identificar possíveis fatores relacionados nos municípios da Macrorregião de Saúde Triângulo do Sul, do estado de Minas Gerais, no período de 2008 a 2012. Métodos: estudo ecológico sobre os coeficientes de mortalidade por Diabetes Mellitus. Foi utilizado o índice de Moran, a série histórica anual, o teste do Qui-Quadrado e a correlação de Pearson. Os resultados da distribuição espacial foram apresentados em um mapa representativo sobre os coeficientes médios de mortalidade. Resultados: a amostra do estudo foi composta pelos 694 casos de óbitos por Diabetes Mellitus, registrados no Sistema de Informação sobre Mortalidade. A mortalidade por Diabetes Mellitus apresentou tendência de aumento no estado de Minas Gerais e na Macrorregião de Triângulo do Sul. O sexo feminino apresentou as taxas mais levadas. Foi encontrada associação entre a mortalidade por sexo em relação à faixa etária e a escolaridade. Conclusões: é relevante que os profissionais da saúde estejam atentos acerca do cenário epidemiológico da mortalidade por Diabetes Mellitus, a fim de fortalecer $\mathrm{o}$ enfrentamento a doença.
\end{abstract}

Palavras-chave: Diabetes Mellitus; Análise Espacial; Epidemiologia; Saúde Coletiva.

\begin{abstract}
Objective: analyze the spatiotemporal pattern of mortality from diabetes mellitus and identify possible factors related in the municipalities of South Triangle Health Macro-Region, state of Minas Gerais, from 2008 to 2012. Method: ecological study of the mortality rates for diabetes mellitus. Moran index, annual historical series, the chi-square and Pearson's correlation was used. The results of the spatial distribution were presented in a representative map on the average mortality rates. Results: the study sample was composed of 694 cases of deaths due to diabetes mellitus, registered in the Mortality Information System. Mortality from diabetes mellitus shows increasing trend in the state of Minas Gerais and in South Triangle Health Macro-Region. The women had the highest rates. Association was found between mortality by sex in terms of age and education. Conclusions: it is important that health professionals are aware about the epidemiological scenario of mortality from diabetes mellitus in order to strengthen the coping the disease.
\end{abstract}

Keywords: Diabetes Mellitus; Spatial Analysis; Epidemiology; Public Health.

\section{RESUMEN}

Objetivo: analizar el patrón espacio-temporal de la mortalidad por diabetes mellitus e identificar posibles factores relacionados, en los municípios de Macro-Salud Triángulo del Sur, en el estado de Minas Gerais, 2008-2012. Método: estudio ecológico de las tasas de mortalidad por diabetes mellitus. Se utilizó el índice de Moran, serie histórica anual, el chi-

\footnotetext{
${ }^{1}$ Enfermeira. Discente do Mestrado em Ambiente e Desenvolvimento, Centro Universitário UNIVATES.

${ }^{2}$ Doutor em Ecologia pela Universidade de São Paulo (USP). Professor Titular da Univates, Centro de Ciências Biológicas e da Saúde.
} 
cuadrado y la correlación de Pearson. Los resultados de la distribución espacial fueron presentados en un mapa representativo de las tasas de mortalidad promedio. Resultados: la muestra del estudio estaba compuesta por 694 casos de muertes debidas a la diabetes mellitus, registrada en el Sistema de Información sobre Mortalidad. La mortalidad por diabetes mellitus muestra la tendencia creciente en el estado de Minas Gerais y en el Macro-Salud Triángulo del Sur. Las mujeres tenían las tasas más altas. Se encontró asociación entre la mortalidad por sexo en cuanto a la edad y la educación. Conclusiones: es importante que los profesionales de la salud son conscientes de la situación epidemiológica de la mortalidad por diabetes mellitus con el fin de fortalecer el afrontamiento de la enfermedad.

Descriptores: Diabetes Mellitus; Análisis Espacial; Epidemiologia; Salud Pública.

\section{INTRODUÇÃO}

As Doenças Crônicas Não

Transmissíveis (DCNT) são apontadas como um problema de saúde pública relevante, devido à sua carga nacional e em função dos prejuízos oriundos, entre eles, aqueles relacionados com a qualidade de vida dos sujeitos e com os gastos elevados para o sistema de saúde. Essas doenças têm como características possuírem múltiplos fatores de risco e estarem relacionadas com deficiências e incapacidades físicas e funcionais nos sujeitos acometidos. ${ }^{1}$

Entre as DCNT, o Diabetes Mellitus (DM) tem apresentado uma tendência de aumento nas taxas de prevalência em nível mundial, trazendo consigo impactos negativos para a qualidade de vida da população portadora da doença. Isso porque o DM, em sua fase inicial, tende a ser assintomático, potencializando a evolução da doença sem a percepção do sujeito. $^{2}$
Dados apontam que no ano de 2013, a prevalência do DM foi de 8,3\% em nível mundial, ocasionando o óbito de cerca de 5,1 milhões de sujeitos com idade entre 20 e 79 anos, sendo que $48 \%$ destes óbitos ocorreram em pessoas com menos de 60 anos de idade, chamando a atenção para o potencial negativo em relação a mortalidade da população em idade produtiva. ${ }^{3}$ No Brasil, os dados indicam que no ano de 2013 a prevalência da doença foi de $9,4 \%$, com mais de 100.000 casos de óbitos decorrentes da doença em sujeitos com idade entre 20 a 79 anos. $^{2}$

Diante do exposto, considera-se relevante compreender o padrão espaçotemporal da mortalidade por DM, a fim de apontar as áreas onde as taxas ocorrem em maior escala, para o planejamento de novas ações e estratégias, e o fortalecimento daquelas que estão em vigor. Este estudo questiona como está configurado o padrão espaço-temporal da mortalidade por DM nos municípios da Macrorregião de Saúde 
Triângulo do Sul, do estado de Minas Gerais, no período de 2008 a 2012?

Com intuito de responder o problema de pesquisa, o objetivo deste estudo foi analisar o padrão espaço-temporal da mortalidade por DM e identificar possíveis fatores relacionados nos municípios da Macrorregião de Saúde Triângulo do Sul, do estado de Minas Gerais, no período de 2008 a 2012.

\section{MÉTODO}

Estudo ecológico, sobre a análise espaço-temporal do conjunto de óbitos por DM ocorridos nos municípios da Macrorregião de Saúde Triângulo do Sul, do estado de Minas Gerais, no período de 2008 a 2012. A Macrorregião de Saúde Triângulo do Sul é uma das 13 Macrorregiões de Saúde do estado de Minas Gerais, localizada ao oeste do estado e ao sul do Triângulo Mineiro. ${ }^{4}$ Esta macrorregião é formada por 27 municípios, área territorial de $29.644 \mathrm{Km}^{2}, 697.812$ habitantes, densidade demográfica de 23,11 Habitantes $/ \mathrm{Km}^{2}$ e PIB de 18.243.782,30. Entre os municípios, o que se destaca pelo maior número populacional é Uberaba, com 295.988 habitantes. ${ }^{5}$

A população em estudo foi composta pelos óbitos por DM dos sujeitos residentes nos 27 municípios que compõe a
Macrorregião de Saúde Triângulo do Sul / Minas Gerais, integrando a amostra de 694 óbitos. Para tanto, o indicador selecionado para a análise foi $o$ coeficiente de mortalidade por DM, discriminado pelas categorias da CID-10 E-10 (diabetes mellitus insulino-dependente) E-11 (diabetes mellitus não-insulinodependente), E-12 (diabetes mellitus relacionado com a desnutrição), E-13 (outros tipos especificados de diabetes mellitus) e E-14 (diabetes mellitus não especificado), relativas ao período de 2008 a 2012, obtidas no Sistema de Informações sobre Mortalidade (SIM), do Sistema de Informática em Saúde do Sistema Único de Saúde (DATASUS). ${ }^{4}$ Os dados foram coletados utilizando as variáveis: ano do óbito e local de residência.

O coeficiente médio de mortalidade de cada município foi calculado utilizando como numerador a média de óbitos por DM do período entre 2008 a 2012 e, como denominador, a população de cada município no ano de $2010^{5}$, escolhido por ser o ano do meio da série histórica analisada e por contemplar os dados do censo demográfico. O resultado foi multiplicado por 100.000 habitantes e utilizado para a distribuição, análise espacial e análise estatística.

Para a compreensão do padrão temporal da mortalidade por DM no estado 
de Minas Gerais, e na Macrorregião Triângulo do Sul, foi analisada a série histórica anual, utilizando o coeficiente de mortalidade anual. O cálculo teve como numerador o número absoluto de óbitos por DM de cada unidade de análise para cada ano e como denominador a população $^{5}$ de cada ano, multiplicado por 100.000 .

Para a análise espacial foi utilizada a análise do Índice de Moran Global (I), que permite a aferição da existência de correlação espacial acerca dos coeficientes médios de mortalidade por DM entre os municípios da Macrorregião Triângulo do Sul. Esse índice varia de -1 a 1 onde resultados próximos a $\quad-1$ indicam correlação inversa, resultados próximos a zero indicam aleatoriedade e valores próximos a 1 indicam correlação espacial direta. $^{6}$

A estatística espacial foi calculada utilizando o método sem peso para a distância entre os vizinhos e considerando a estratégia por contiguidade, a qual aponta como vizinhos os municípios que dividem fronteira. ${ }^{6}$ A malha cartográfica utilizada foi coletada no sítio do DATASUS. ${ }^{4}$

A variável dependente do estudo foi o coeficiente de mortalidade por DM e as variáveis independentes foram os municípios que fazem parte da Macrorregião Triângulo do Sul e as variáveis demográficas, socioeconômicas e de cobertura da Atenção Básica. Essas variáveis são apresentadas por: sexo, faixa etária, cor / raça, renda per capita domiciliar municipal em 2010, proporção de analfabetismo em 2010 (na população de $\geq 15$ anos de idade) e proporção média de cobertura da Atenção Básica (no período de 2008 a 2012) de cada município da Macrorregião Triângulo do Sul (indicadores da atenção básica). As variáveis renda per capita domiciliar municipal e proporção de analfabetismo em 2010 (na população de $\geq 15$ anos de idade) são oriundas do $\mathrm{IBGE}^{5}$, enquanto que as demais foram coletadas no sítio do DATASUS. ${ }^{4}$

Os resultados descritivos dos dados sobre a mortalidade por DM, em cada categoria de faixa etária e cor / raça estratificada por sexo, foram apresentados em números absolutos e em frequências. Os mesmos dados foram submetidos à análise pelo teste Qui-quadrado, a fim de avaliar a associação entre sexo e faixa etária, cor / raça e escolaridade. A correlação de Pearson foi utilizada para analisar a correlação entre a mortalidade por DM, em cada município, com as demais variáveis independentes. Foi considerada a significância de $\leq 0,05$.

A tabulação dos dados e a transformação dos coeficientes foi 
elaborada no software TabWin 32, a distribuição e a análise espacial foram desenvolvidas no software TerraView 4.2.2 e a análise estatística foi realizada no software SPSS 20. A presente pesquisa não apresenta implicações éticas, pois utilizou dados de domínio público. Desta forma, não foi necessária a submissão ao Comitê de Ética em Pesquisa.

\section{RESULTADOS}

Os resultados descritivos apontam 694 óbitos por DM ocorridos nos municípios que fazem parte da
Macrorregião de Saúde Triângulo do Sul no período estudado. Destes, $54 \%$ fazem parte do sexo feminino, $26 \%$ contemplam a faixa etária dos 70 aos 79 anos, sendo esta a faixa etária com maior número de óbitos, e $84 \%$ contemplam a CID-10 E14.

O resultado da série histórica anual aponta para uma tendência de aumento na mortalidade por DM para o estado de Minas Gerais e para a Macrorregião de Triângulo do Sul, no período analisado (Gráfico 1). Para o estado de Minas Gerais, o aumento representou $22 \%$ e para a Macrorregião de Triângulo do Sul o aumento foi de $43 \%$.

Gráfico 1 - Coeficiente de mortalidade por Diabetes Mellitus na Macrorregião de Saúde Triângulo do Sul / Minas Gerais, no período de 2008 a 2012.

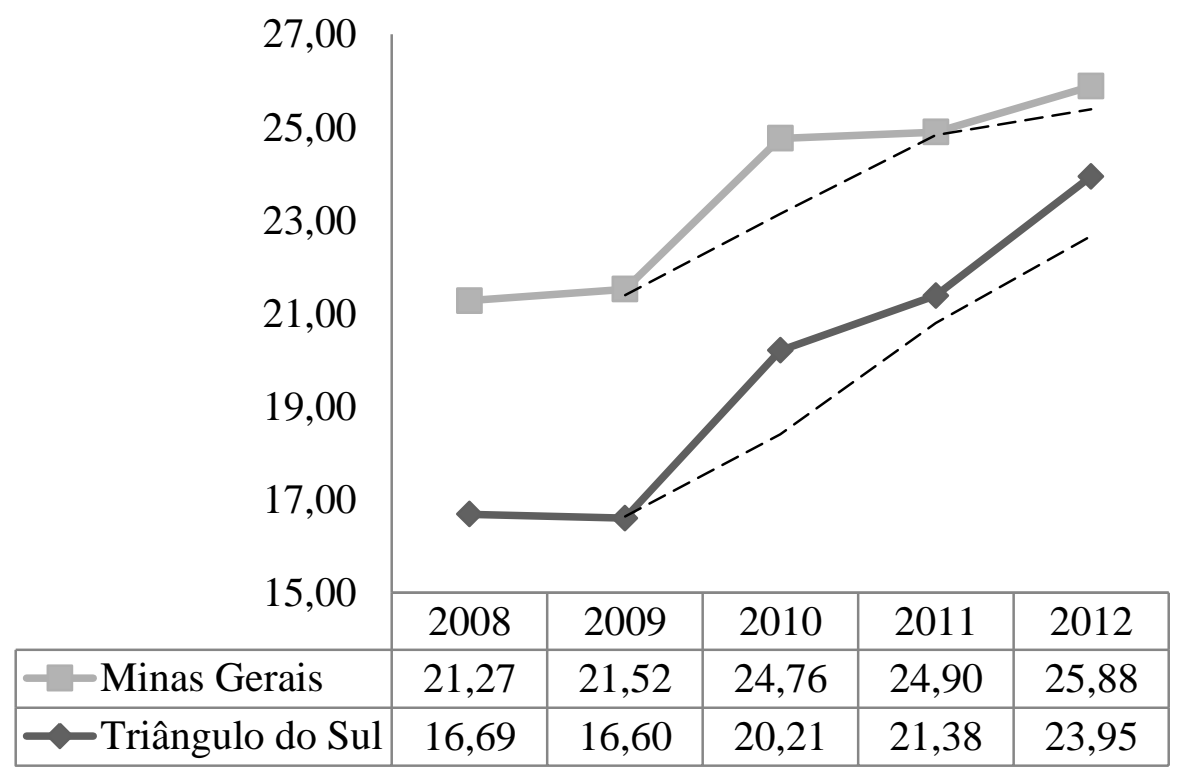

O resultado da distribuição espacial permite visualizar de forma geral qual é o padrão espacial da mortalidade por DM na Macrorregião Triângulo do Sul. Percebe-se que o município de Água Comprida, seguido dos municípios de Araxá, de Ibiá e de Pratinha, é o que apresenta coeficiente mais elevado para a variável de desfecho. O Índice de Moran Global apresentou 
valores próximos a zero e não significativos (Figura 1).

Figura 1 - Distribuição espacial do coeficiente médio de mortalidade por Diabetes Mellitus na Macrorregião de Saúde Triângulo do Sul / Minas Gerais, 2008 a 2012.

$$
I=0.0450847(\mathrm{p}=0.37)
$$

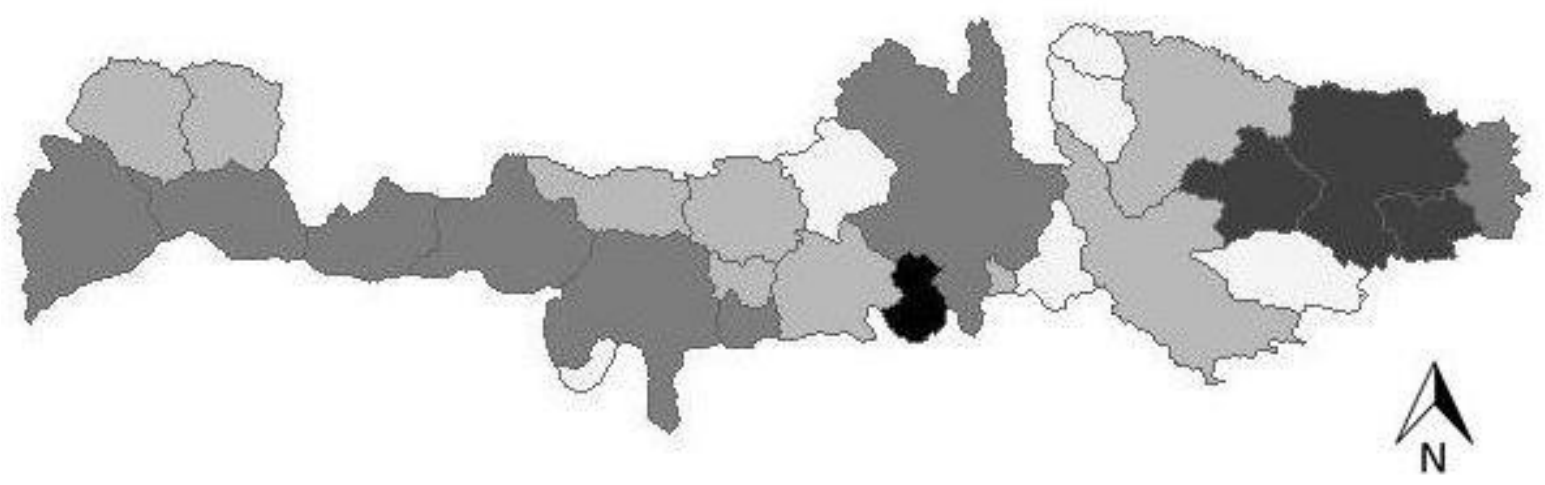

Diabetes_Mellitus

$-0.01 \sim 7.90$

$7.90 \sim 15.80$

$15.80 \sim 23.71$

$23.71 \sim 31.61$

$31.61 \sim 39.52$

O coeficiente médio de mortalidade para a Macrorregião Triângulo do Sul, no período de estudo, foi de $19,76 \%$. Nesta perspectiva, os municípios que apresentaram valores mais críticos, estando acima da média da Macrorregião, foram Água Comprida (39,51), Araxá $(31,17)$, Ibiá (31,01), Pratinha (24,50), Iturama $(22,64)$, Planura $(21,19)$ e Uberaba $(19,80)$.

Os resultados apontam associação entre sexo e as variáveis faixa etária e escolaridade. Existe uma tendência entre o sexo masculino com a faixa etária dos 40 aos 59 anos, e o sexo feminino com a faixa etária dos 60 aos $\geq 80$ anos. Em relação à escolaridade, os dados indicam tendência maior entre o sexo feminino e a baixa escolaridade (0 a 3 anos de estudo) e o sexo masculino com escolaridade entre 4 a $\geq 12$ anos de estudo (tabela 1 ).

Tabela 1 - Óbitos por Diabetes Mellitus por sexo, faixa etária e cor / raça na Macrorregião de Saúde Triângulo do Sul / Minas Gerais, 2008 a 2012.

\begin{tabular}{ccc}
\hline Masculino & Feminino & $\mathbf{p}^{*}$ \\
$\mathrm{~N}(\%)$ & $\mathrm{N}(\%)$ & \\
\hline
\end{tabular}




\begin{tabular}{lccc}
\hline Faixa Etária & $31(72 \%)$ & $12(28 \%)$ & $<0.0001$ \\
40 a 49 anos & $60(55 \%)$ & $49(45 \%)$ & \\
50 a 59 anos & $79(49 \%)$ & $82(51 \%)$ & \\
60 a 69 anos & $77(43 \%)$ & $104(57 \%)$ & 0.0001 \\
70 a 79 anos & $58(34 \%)$ & $115(66 \%)$ & \\
$\geq 80$ anos & & & \\
Escolaridade & $21(27 \%)$ & $57(73 \%)$ & \\
Nenhuma & $85(46 \%)$ & $99(54 \%)$ & \\
1 a 3 anos & $54(61 \%)$ & $35(39 \%)$ & \\
4 a 7 anos & $24(53 \%)$ & $21(47 \%)$ & \\
8 a 11 anos & $15(65 \%)$ & $8(35 \%)$ & \\
$\geq 12$ anos & & & \\
Cor / raça & $197(45 \%)$ & $243(55 \%)$ & \\
Branca & $41(47 \%)$ & $46(53 \%)$ & \\
Preta & $49(58 \%)$ & $36(42 \%)$ & \\
Parda &
\end{tabular}

* Teste do Qui-quadrado (excluídas as informações ignoradas).

Não foi encontrada associação entre sexo e cor / raça, porém os resultados descritivos apontam predominância do sexo feminino entre a população de cor / raça branca e preta, acometida pelo DM, e do sexo masculino com a população de cor / raça parda (Tabela 1). Os resultados da análise da correlação de Pearson apontam que não existe significância entre a mortalidade por DM e a taxa de analfabetismo, a renda per capita domiciliar e a proporção de cobertura da Atenção Básica.

\section{DISCUSSÃO}

Os resultados apontam que a mortalidade por DM esteve presente em maior frequência entre a população do sexo feminino. Este dado tem sido indicado também em outros estudos ${ }^{7-9}$, e demonstra que esse padrão segue uma tendência visualizada também em pesquisas desenvolvidas em outros países.

A mortalidade por DM se mostrou mais predominante entre a população com idade entre os 70 aos 79 anos. Um estudo realizado com idosos atendidos pela Estratégia Saúde da Família (ESF), em um município de Minas Gerais, apontou que na população idosa a mais acometida pelo DM foi a da faixa etária entre os 70 aos 79 anos. Nesta perspectiva, o mesmo estudo aborda a importância dos profissionais da saúde estarem preparados para subsidiar ações de promoção à saúde para a população desta faixa etária. ${ }^{10}$ 
Entre as categorias da CID-10, analisadas neste estudo, a que apontou maior frequência de mortalidade foi a categoria E-14, que contempla o DM não especificado. Isso pode trazer um viés para a análise da mortalidade em relação ao tipo de DM. De qualquer forma, os dados coletados no SIM sobre a mortalidade por DM não deixam de ser relevante, uma vez que possibilitam a compreensão do padrão de mortalidade pela doença. Além disso, a American Diabetes Association ${ }^{11}$ aborda que em torno de $90 \%$ dos diabéticos possuem o DM não-insulino-dependente (categorizado pela CID-10 E11).

A tendência temporal apontou aumento da mortalidade por DM, tanto na Macrorregião de Triângulo do Sul quanto no estado de Minas Gerais, no período de 2008 a 2012. O aumento da mortalidade por DM também é descrito em outros estudos. $^{8,9,12}$

O Plano de Ações Estratégicas para o Enfrentamento das Doenças Crônicas Não Transmissíveis no Brasil 2011-2022 estabeleceu a meta de redução da mortalidade prematura por DCNT em $2 \%$ ao ano. ${ }^{13}$ Porém, um estudo apontou que o estado de Minas Gerais possui cenário desfavorável para o cumprimento da meta de redução da mortalidade prematura no contexto do DM. ${ }^{14}$
Nesta perspectiva, percebe-se a importância do fortalecimento das estratégias de enfrentamento ao DM já existentes, entre elas o Sistema de Cadastramento e Acompanhamento de Hipertensos e Diabéticos (SisHiperdia) ${ }^{15}$, que tem potencial para subsidiar as ações de promoção à saúde no contexto do DM, através da identificação do problema e do acompanhamento dos diabéticos.

O presente estudo não apontou correlação espacial significativa para a taxa de mortalidade por DM, entre os municípios da região de saúde de Triângulo do Sul. Porém, um estudo que analisou a correlação espacial da prevalência de DM, entre os municípios do estado de Minas Gerais, apontou para Índices de Moran positivos e significativos, chamando a atenção para um cluster localizado no sul do estado, que possuía taxas elevadas para o DM e também para a HAS. ${ }^{16}$

A distribuição espacial possibilitou apontar os municípios, da Macrorregião Triângulo do Sul, que apresentaram as taxas mais críticas de mortalidade por DM. Este resultado é importante para que sejam (re)pensadas ações em saúde e estratégias de intervenção nos locais mais vulneráveis e que necessitam de maiores intervenções. Estudos tratam da importância da abordagem espacial para a análise do perfil 
epidemiológico da mortalidade por DM. $^{7,9,12}$

Os dados apontam que existe uma tendência preocupante, a frequência na mortalidade por DM em homens com idade entre 40 aos 59 anos, em relação ao sexo feminino. Segundo a Política Nacional de Atenção Integral à Saúde do Homem, os homens procuram com menos frequência os serviços de saúde, em relação às mulheres, acarretando maior mortalidade deles em idade produtiva e por causas evitáveis. ${ }^{17}$

Neste estudo, não foi encontrada correlação significativa entre a mortalidade por DM e as variáveis taxa de analfabetismo, renda per capita domiciliar e proporção de cobertura da Atenção Básica. Um estudo, que também utilizou estas três variáveis para analisar a correlação entre elas com a mortalidade por doenças cardiovasculares, apontou correlação inversa para a variável renda per capita em municípios, ao sul do País, com mais de 15.000 habitantes. ${ }^{18}$

Os números absolutos indicam que a escolaridade entre 1 a 3 anos de estudo é a que abrange o maior número de óbitos por DM, com frequência maior entre as mulheres. Nesta perspectiva, a literatura apresenta um estudo sobre o letramento em saúde em relação ao Diabetes Mellitus tipo 2 (DM2 ou Diabetes Mellitus não-insulino- dependente), onde a maior parte dos sujeitos que compõe a amostra $(65,9 \%)$ apresentaram letramento em saúde inadequado. $^{19}$

A alfabetização já foi descrita anteriormente enquanto fator relevante para o enfrentamento ao $\mathrm{DM}^{20}$. $\mathrm{O}$ analfabetismo funcional em saúde é um grande desafio para os profissionais em Saúde, principalmente aos Enfermeiros, que precisam encontrar estratégias que possibilitem esclarecer aos usuários dos serviços de saúde os cuidados necessários em relação ao $\mathrm{DM}$, a fim de controlar a doença e prevenir as complicações oriundas. ${ }^{19}$

\section{CONCLUSÕES}

É relevante considerar algumas limitações deste estudo. Primeiramente, os dados são oriundos de um banco de dados secundário, que pode possuir problemas em relação à subnotificação e ao subregistro. Além disso, os dados são agregados, acarretando a dificuldade de controlar os fatores de confusão. Ademais, os óbitos decorrentes do DM podem ter sua causa identificada como sendo por outras doenças, como por exemplo, as doenças renais, a HAS e outras doenças cardiovasculares. Entretanto, é pertinente considerar a importância do SIM como 
instrumento facilitador para a elaboração de indicadores em saúde.

A mortalidade por DM tem apresentado tendência de aumento, durante o período de 2008 a 2012, no estado de Minas Gerais e na Macrorregião de Saúde de Triângulo do Sul. A distribuição espacial possibilitou apontar os municípios onde as taxas apresentam valores mais elevados.

Os resultados indicam associação entre sexo e faixa etária e escolaridade no contexto da mortalidade por DM. Apontando, em especial, a tendência da mortalidade precoce para a população do sexo masculino. Uma hipótese para esse problema pode estar no fato dos homens

\section{REFERÊNCIAS}

1. Ministério da Saúde (BR). A vigilância, o controle e a prevenção das doenças crônicas não-transmissíveis: DCNT no contexto do Sistema Único de Saúde brasileiro. Brasília: Ministério da Saúde; Organização Pan-Americana da Saúde, 2005.

2. International Diabetes Federation (IDF) [internet]. IDF Diabetes Atlas Update, 2013. [Acesso em 20/Nov/2015] Disponível em: http://www.idf.org/diabetesatlas/download -book.

3. International Diabetes Federation (IDF) [internet]. Key Findings 2014. Bélgica: IDF; 2014. [Acesso em 20/Nov/2015] Disponível em: http://www.idf.org/sites/default/files/Atlasposter-2014_EN.pdf. procurarem com menos frequência os serviços de saúde. Isso pode contribuir para o desconhecimento sobre $\mathrm{o}$ acometimento pelo DM, favorecendo a evolução da doença, dos agravos associados e aumentando as chances de mortalidade.

Diante disso, é relevante que os profissionais da saúde estejam atentos, em especial os Enfermeiros, a fim de propor estratégias com intuito de orientar os usuários dos serviços de saúde sobre o DM, e sobre as ações de prevenção e de manejo, buscando com isso a promoção da saúde, a qualidade de vida dos sujeitos e a diminuição de gastos oriundos para o sistema de saúde.

4. DATASUS [Internet]. Brasília: Ministério da saúde (BR). [Acesso em 15/Nov/2015] Disponível em: http://www.datasus.gov.br/datasus/datasus. php.

5. Instituto Brasileiro de Geografia e Estatística (IBGE) [internet]. [Acesso em 20/Nov/2015] Disponível em: http://www.ibge.gov.br.

6. Ministério da Saúde (BR). Introdução à estatística espacial para saúde pública. Brasília: Ministério da Saúde; 2007.

7. Zhou M, Astell-Burt T, Yin P, Feng X, Page A, Liu Y, et al. Spatiotemporal variation in diabetes mortality in China: multilevel evidence from 2006 and 2012. BMC Public Health. 2015; 15(633): 1 - 10.

8. Santana P, Costa C, Loureiro A, Raposo J, Boavida JM. Geografias da Diabetes Mellitus em Portugal: Como as Condições do Contexto Influenciam o Risco de 
Morrer. Acta Med Port. 2014; 27(3): 309317.

9. Sánchez-Barriga JJ. Mortality trends from diabetes mellitus in the seven socioeconomic regions of Mexico, 20002007. Rev Panam Salud Publica. 2010; 28(5): 368-375.

10. Pimenta FB, Pinho L, Silveira MF, Botelho ACC. Fatores associados a doenças crônicas em idosos atendidos pela Estratégia de Saúde da Família. Cien Saude Colet. 2015; Ago; 20(8): 2489-2498.

11. American Diabetes Association. Diagnosis and classification diabetes mellitus. Diabetes Care 2012; (35Supl. 1): S64-S71.

12. Dávila-Cervantes CA, Pardo Montaño AM. Diabetes mellitus: Contribution to changes in the life expectancy in Mexico 1990, 2000, and 2010. Rev. salud pública. 2014; 16(6): 910-923.

13. Ministério da Saúde. Secretaria de Vigilância em Saúde (BR). Plano de ações estratégicas para o enfrentamento das doenças crônicas não transmissíveis (DCNT) no Brasil 2011-2022. Brasília: Ministério da Saúde, 2011.

14. Alves CG, Morais Neto OL. Trends in premature mortality due to chronic noncommunicable diseases in Brazilian federal units. Cien Saude Colet. 2015; 20(3): 641654.

15. Ministério da Saúde (BR). Relatório de gestão 1998-2002. Brasília: Ministério da Saúde, 2002.
16. Pinto ESO, Santos GR, Oliveira FLP. Análise espaço-temporal aplicada às ocorrências de hipertensão e diabetes nos municípios do estado de Minas Gerais. Rev. Bras. Biom. 2014; 32(2): 238 - 266.

17. Ministério da Saúde. Secretaria de Atenção à Saúde (BR). Política Nacional de Atenção Integral à Saúde do Homem: princípios e diretrizes. Brasília: Ministério da Saúde; 2009.

18. Medeiros CRG, Meneghel SN, Gerhardt TE. Desigualdades na mortalidade por doenças cardiovasculares em pequenos municípios. Cien Saude Colet. 2012; 17(11): 2953-2962.

19. Sampaio HAC, Carioca AAF, Sabry MOD, Santos PM, Coelho MAM, Passamai MPB. Letramento em saúde de diabéticos tipo 2 - fatores associados e controle glicêmico. Cien Saude Colet. 2015; 20(3): 865-874.

20. Nava S, Carreno I, Rempel C, Schwingel G, Pissaia LF, Belé P. Perfil epidemiológico da hipertensão e diabetes em mulheres. [online]. Rev Enferm Atenção Saúde, 2015. [acesso 20/Nov/15]; 4(1):42-54. Disponível em: http://www.uftm.edu.br/revistaeletronica/in dex.php/enfer/article/view/1262/1133.

Recebido em 29/08/2016

Aprovado em: 15/12/2016

Publicado em: 29/12/2016 